\title{
Quantum Forces from Dark Matter and Where to Find Them
}

\author{
Sylvain Fichet \\ ICTP-SAIFR and IFT-UNESP, Rua Dr. Bento Teobaldo Ferraz, 271, São Paulo, Brazil
}

(Received 19 June 2017; revised manuscript received 5 January 2018; published 27 March 2018)

\begin{abstract}
We observe that sub-GeV dark matter (DM) induces Casimir-Polder forces between nucleons that can be accessed by experiments from nuclear to molecular scales. We calculate the nucleon-nucleon potentials arising in the DM effective theory and note that their main features are fixed by dimensional analysis and the optical theorem. Molecular spectroscopy and neutron scattering turn out be DM search experiments and are found to be complementary to nucleon-based DM direct detection. Existing data set limits on DM with mass up to $\sim 3-50 \mathrm{MeV}$ and with effective interaction up to the $O(10-100) \mathrm{MeV}$ scale, constraining a region typically difficult to reach for other experiments.
\end{abstract}

DOI: 10.1103/PhysRevLett.120.131801

Introduction.-A body of evidence suggests that our Universe is filled with an unknown dark matter (DM), which may be a new kind of particle lying beyond the standard model (SM) of particle physics. What do we know about this putative dark particle? Apart from its weak interaction with photons, very little is known about its properties, including mass, spin, and couplings. Importantly, a robust lower bound is set from structure formation in the Universe: galaxy formation implies the dark particle mass should satisfy $m \gtrsim 2 \mathrm{keV}[1-3]$ to limit its free-streaming length.

Although direct detection experiments have reached impressive sensitivity above the $\mathrm{GeV}$ mass scale, the dark particle remains so far elusive [4]. For masses below the $\mathrm{GeV}$, direct detection methods lose sensitivity because nuclear recoil becomes too soft to be detected. At the LHC, monojet searches could be sensitive to a dark particle below the $\mathrm{GeV}$ if it has a contact interaction with the SM particles. However, when the interaction between the DM and SM is resolved at the LHC, the sensitivity is expected to vanish when the scale of this "portal" becomes too low. For instance, for mediation via a $Z^{\prime}$ particle, the sensitivity vanishes below roughly $O(10-100) \mathrm{GeV}$ [5].

As the two most direct search techniques-scattering on nucleons and monojets-are inefficient when DM is sub$\mathrm{GeV}$ and the portal scale is light (forming thus a "light dark sector"), other experiments need to be devised. Cosmological and astrophysical constraints can of course play a role, but are somewhat indirect an depend on many assumptions; hence, more direct searches for light dark particles are certainly needed. As a matter of fact, an increasing number of ideas are being proposed to search for sub-GeV DM,

Published by the American Physical Society under the terms of the Creative Commons Attribution 4.0 International license. Further distribution of this work must maintain attribution to the author(s) and the published article's title, journal citation, and DOI. Funded by SCOAP ${ }^{3}$. including semiconductor [6-9], superconductor [10,11], and superfluid [12,13] targets, carbon structures [14,15], crystals [16,17], scintillators [18], electron scattering or bremsstrahlung in conventional detectors [19-21], neutrino fixed target experiments [22-29], and the SHiP proposal [30]. All these proposals rely on dark particles on the mass shell.

In this Letter, we take a different approach by considering a phenomenon induced by "virtual" dark particles (see also [31]). We point out that, whenever sub-GeV dark matter couples to nucleons, it induces Casimir-Polder forces between them. The $m \gtrsim 2 \mathrm{keV}$ bound from structure formation corresponds then to a maximum scale of $\sim 1 \AA$ for the force range, implying that these DM-induced forces can be active up to molecular scales. We will see that molecular and atomic precision spectroscopy as well as neutron scattering experiments are sensitive to such forces. Existing data from these research fields will be exploited in this Letter to obtain new limits on sub-GeV DM. In the following, we will refer to the DM-induced Casimir-Polder forces simply as "DM forces".

The focus in this Letter is on DM that interacts with nucleons. Interactions with electrons could also be studied, although they are already constrained by $e^{+} e^{-}$collisions [32]. Our approach relies on virtual dark particles; henc The DM forces between electrons and nucleons could be studied by analyzing King plots from isotope shift spectroscopy, a technique recently proposed in [33-36].

Our approach relies on virtual dark particles; hence, all our results apply whether or not the dark particle is stable; an agnostic viewpoint is taken in Ref. [37]. Yet, the implications for dark matter deserve special attention given the profusion of experimental and theoretical activities in this area. This Letter is thus focused on the implications for DM searches and for some predictive DM cosmological scenarios.

Casimir-Polder forces from dark matter effective theory.Whenever DM interacts with light quarks or with gluons, it couples to nucleons below the QCD scale. We use an 
effective field theory (EFT) to describe the DM interactions with nucleons. Our most general results for DM forces are given here, but more details on EFT and calculation are available in Ref. [37].

In the limit of unpolarized nonrelativistic nucleons, only the interactions involving $\bar{N} N, \bar{N} \gamma^{\mu} N$ are relevant. The DM particle is noted $\phi, \chi$, and $X$ for particles of spin $0,1 / 2$, and 1 , respectively-either self-conjugate or not. Results will be presented for a representative subset of interactions for DM particles of each spin,

$\mathcal{O}_{a}^{0}=\frac{1}{\Lambda} \bar{N} N|\phi|^{2}, \quad \mathcal{O}_{a}^{1 / 2}=\frac{1}{\Lambda^{2}} \bar{N} N \bar{\chi} \chi$,

$\mathcal{O}_{b}^{0}=\frac{1}{\Lambda^{2}} \bar{N} \gamma^{\mu} N \phi^{*} i \stackrel{\leftrightarrow}{\partial}_{\mu} \phi, \quad \mathcal{O}_{b}^{1 / 2}=\frac{1}{\Lambda^{2}} \bar{N} \gamma^{\mu} N \bar{\chi} \gamma^{\mu} \chi$,

$\mathcal{O}_{c}^{0}=\frac{1}{\Lambda^{3}} \bar{N} N \partial^{\mu} \phi^{*} \partial_{\mu} \phi, \quad \mathcal{O}_{c}^{1 / 2}=\frac{1}{\Lambda^{2}} \bar{N} \gamma^{\mu} N \bar{\chi} \gamma^{\mu} \gamma^{5} \chi$,

$\mathcal{O}_{a}^{1}=\frac{m^{2}}{\Lambda^{3}} \bar{N} N\left|X^{\mu}+\partial^{\mu} \pi\right|^{2}$,

$\mathcal{O}_{b}^{1}=\frac{1}{\Lambda^{2}} 2 \bar{N} \gamma^{\mu} N \operatorname{Im}\left[X_{\mu \nu}^{*} X^{\nu}+\partial^{\nu}\left(X_{\nu} X_{\mu}^{*}\right)+\partial^{\mu} \bar{c} c^{*}\right]$,

$\mathcal{O}_{c}^{1}=\frac{1}{\Lambda^{3}} \bar{N} N\left|X^{\mu \nu}\right|^{2}, \quad \mathcal{O}_{d}^{1}=\frac{1}{\Lambda^{3}} \bar{N} N X^{\mu \nu} \tilde{X}^{\mu \nu}$,

where $\stackrel{\leftrightarrow}{\partial}=\vec{\partial}-\overleftarrow{\partial}$, and $2 \operatorname{Im}\left(X_{\mu \nu}^{*} X^{\nu}\right)+\cdots$ corresponds to the gauge current for $X . \pi$ and $c, \bar{c}$ are, respectively, the Goldstone and ghosts accompanying $X$. The $\mathcal{O}_{b}^{s}$ operators involve gauge currents of DM, which vanish if DM is selfconjugate (real scalar or vector, Majorana fermion). For simplicity, we will assume a universal coupling to protons

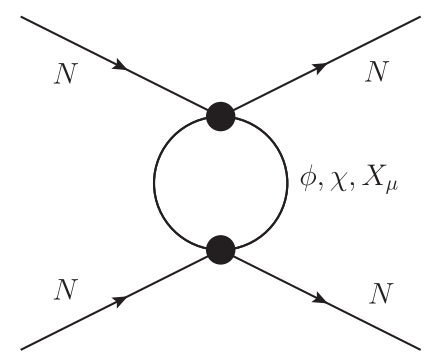

FIG. 1. The exchange of two dark particles inducing a force between the nucleons.

and neutrons-all our results are easily generalized for nonuniversal couplings to nucleons.

The effective interactions of Eq. (1) induce four-nucleon interactions when closing the DM loop, suggesting to search for DM in nucleon interactions. The diagram being a loop amplitude in the EFT, local four-nucleon interactions are in principle also present [38] that depend on the UV completion of the theory (such as heavy mediators or intrinsic polarizability [31]). However, the experimental results we will use are by design fully or nearly independent of these local terms and are thus perfectly appropriate to specifically target a light dark particle.

The force between nucleons induced by the diagram in Fig. 1 is obtained by taking the nonrelativistic limit of the amplitude, taking the 3D Fourier transform, continuing in the complex plane, and integrating over a branch cut. The subsequent potentials are given by modified Bessel functions evaluated at $2 m r, K_{i}(2 m r) \equiv K_{i}$. The operators of Eq. (1) give the DM forces

$$
\begin{aligned}
\left(V_{a}^{0},\right. & \left.V_{b}^{0}, V_{c}^{0}, V_{a}^{1 / 2}, V_{b}^{1 / 2}, V_{c}^{1 / 2}, V_{a}^{1}, V_{b}^{1}, V_{c}^{1}, V_{d}^{1}\right) \\
= & \frac{1}{32 \pi^{3} r} \times\left\{-2^{\eta} \frac{\left(C_{a}^{0}\right)^{2}}{\Lambda^{2}} \frac{m}{r} K_{1}, \eta \frac{4\left(C_{b}^{0}\right)^{2}}{\Lambda^{4}} \frac{m^{2}}{r^{2}} K_{2},-2^{\eta} \frac{\left(C_{c}^{0}\right)^{2}}{\Lambda^{6}}\left[\left(\frac{30 m^{2}}{r^{4}}+\frac{6 m^{4}}{r^{2}}\right) K_{2}+\left(\frac{15 m^{3}}{r^{3}}+\frac{m^{5}}{r}\right) K_{1}\right],\right. \\
& -2^{\eta} \frac{12\left(C_{a}^{1 / 2}\right)^{2}}{\Lambda^{4}} \frac{m^{2}}{r^{2}} K_{2}, \eta \frac{16\left(C_{b}^{1 / 2}\right)^{2} m^{2}}{\Lambda^{4}}\left(\frac{1}{r^{2}} K_{2}+\frac{m}{r} K_{1}\right), 2^{\eta} \frac{8\left(C_{c}^{1 / 2}\right)^{2}}{\Lambda^{4}} \frac{m^{2}}{r^{2}} K_{2},-2^{\eta} \frac{\left(C_{a}^{1}\right)^{2}}{\Lambda^{6}}\left[\left(\frac{15 m^{2}}{r^{4}}+\frac{3 m^{4}}{r^{2}}\right) K_{3}\right], \\
& \eta \frac{\left(C_{b}^{1}\right)^{2}}{\Lambda^{4}}\left(\frac{40 m^{2}}{r^{2}} K_{2}+\frac{36 m^{3}}{r} K_{1}\right),-2^{\eta} \frac{12\left(C_{c}^{1}\right)^{2}}{\Lambda^{6}}\left[\left(\frac{20 m^{2}}{r^{4}}+\frac{4 m^{4}}{r^{2}}\right) K_{2}+\left(\frac{10 m^{3}}{r^{3}}+\frac{m^{4}}{r^{4}}\right) K_{1}\right], \\
& \left.-2^{\eta} \frac{24\left(C_{d}^{1}\right)^{2}}{\Lambda^{6}}\left(\frac{2 m^{4}}{r^{2}} K_{3}+\frac{5 m^{3}}{r^{3}} K_{2}\right)\right\} .
\end{aligned}
$$

The forces from the $\mathcal{O}_{a, c}^{s}$ operators ("scalar channel") are attractive and those from the $\mathcal{O}_{b}^{s}$ operators ("vector channel") are repulsive.

The main features of these forces can be understood using both dimensional analysis and the optical theorem applied to the diagram of Fig. 1. First, the optical theorem dictates the sign of the discontinuity over the branch cut and thus the sign of the potentials. Second, the short-distance behavior $\left(r^{-3}, r^{-5}, r^{-7}, r^{-5}, r^{-5}, r^{-5}, r^{-7}, r^{-5}, r^{-7}, r^{-7}\right)$ of Eq. (2) is dictated by dimensional analysis. Third, using the optical theorem, the long-distance behavior $e^{-2 m r}\left(r^{-5 / 2}\right.$, $\left.r^{-7 / 2}, r^{-5 / 2}, r^{-7 / 2}, r^{-5 / 2}, r^{-7 / 2}, r^{-5 / 2}, r^{-5 / 2}, r^{-5 / 2}, r^{-7 / 2}\right)$ is related to velocity suppression of the $\bar{N} N \leftrightarrow \chi \bar{\chi}$ amplitude at $s \sim 4 m^{2}$ (see details in [37]).

Dark matter bounds from molecular spectroscopy.Impressive progress on both the experimental [39-46] and 
theoretical [47-58] sides of precision molecular spectroscopy has been accomplished recently, opening the possibility of testing new physics below the angstrom scale using transition frequencies of well-understood simple molecular systems. Certain of these results have recently been used to bound short-distance modifications of gravity; see Refs. [59-62].

The most relevant systems for which both precise measurements and predictions are available are the hydrogen molecule $\mathrm{H}_{2}$, the molecular hydrogen-deuterium ion $\mathrm{HD}^{+}$, and muonic molecular deuterium ion $d d \mu^{+}$, where $d$ is the deuteron. This last system is exotic in the sense that a heavy particle (the muon) substitutes an electron. As a result, the internuclear distance is reduced, providing a sensitivity to forces of shorter range and thus to heavier dark particles.

The presence of the DM force shifts the energy levels by $\Delta E=\int d^{3} \mathbf{r} \Psi^{*}(r) V(r) \Psi(r)$ at first order in perturbation theory. We compute these energy shifts for the transitions between the $(\nu=1, J=0)-(\nu=0, J=0)$ states for $\mathrm{H}_{2}$, $(\nu=4, J=3)-(\nu=0, J=2)$ of $\mathrm{HD}^{+}$, and the binding energy of the $(\nu=1, J=0)$ state of $d d \mu^{+}$using the wave functions given in $[60,61,63]$, with $\nu$ and $J$ being, respectively, the rotational and vibrational quantum numbers. The average internuclear distances for the quantum states considered are, respectively, $\sim 1 \AA$ for $\mathrm{H}_{2}$ and $\mathrm{HD}^{+}$, and $\sim 0.005-0.08 \AA$ for $d d \mu^{+}$. Bounds on the DM forces are obtained using combined uncertainties of, respectively, $3.9 \mathrm{neV}$ [59,61], $0.33 \mathrm{neV}$ [59], and $\delta E=0.7 \mathrm{meV}$ [60]. For each observable, the experimental uncertainty is slightly larger than the theoretical one, at most by an order of magnitude [64]. Therefore, progress on both experimental and theory sides would be needed in order to improve the sensitivity of these molecular observables.

The lower bounds obtained on $\Lambda$ and $m$ are typically on the order of $10-100$ and 3-50 MeV, respectively. It turns out that limits from $d d \mu^{+}$are the most stringent on both $\Lambda$ and $m$. Sample results are shown in Figs. 2 and 3.

Dark matter bounds from neutron scattering.-Progress in measuring the scattering of cold neutrons on nuclei has recently been made and used to put bounds on shortdistance modified gravity [74-81]. The cold neutron scattering cross section can be measured at zero angle by "optical" methods, at nonzero angles using Bragg diffraction, or over all angles by the "transmission" method, then giving the total cross section [82].

In the following, we adapt the analyses of [80] to the DM case. At low energies, the standard neutron-nuclei interaction is a contact one. New physics can induce both contact and noncontact contributions, and it is convenient to introduce the scattering length $\sqrt{\sigma(\mathbf{q}) / 4 \pi} \equiv l(\mathbf{q})=l_{\text {std }}^{C}+l_{\mathrm{NP}}^{C}+$ $l_{\mathrm{NP}}^{\mathrm{NC}}(\mathbf{q})$, where the $l_{\text {std }}^{C}$ and $l_{\mathrm{NP}}^{C}$ terms are independent of momentum transfer q. A convenient way to look for an anomalous interaction is to search for $l_{\mathrm{NP}}^{\mathrm{NC}}(\mathbf{q})$ by comparing
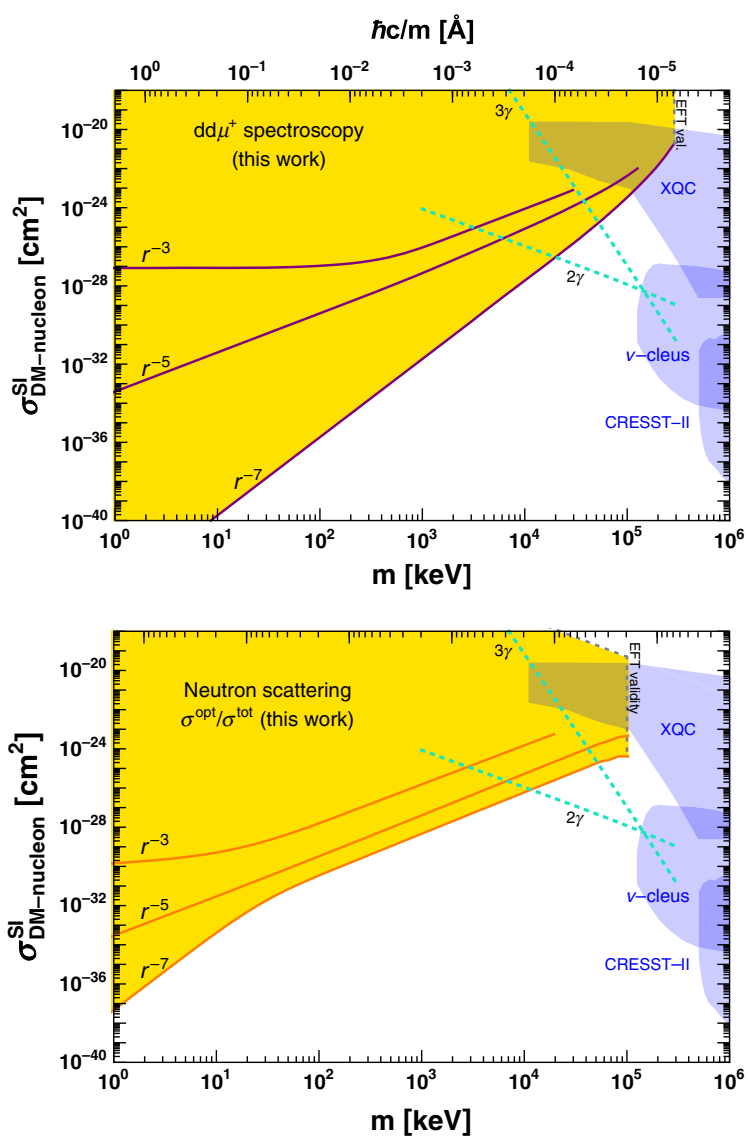

FIG. 2. Leading exclusion regions from DM forces translated into the $\sigma^{\mathrm{SI}}-m$ plane of direct detection. Exclusion regions from a sample of forces with $r^{-3}, r^{-5}$, and $r^{-7}$ short-distance behavior are shown. Direct detection bounds labeled XQC, $\nu$-cleus, and CRESST-II are, respectively, from Refs. [65-67]. Dotted lines correspond to $\Omega h^{2}=0.112$ with thermal freeze-out of Dirac DM $\chi \bar{\chi} \rightarrow 2 \gamma, 3 \gamma$ pion-driven annihilations.

the scattering length obtained by different methods: $l_{\text {Bragg }} / l_{\text {opt }}$ and $l_{\text {tot }} / l_{\text {opt }}$. This approach eliminates $l_{\text {std }}^{C}$, but also $l_{\mathrm{NP}}^{C}$, and is therefore only sensitive to the nonlocal part of the scattering potential, which corresponds to the DM force. Sample results from $l_{\text {Bragg }} / l_{\text {opt }}$ and $l_{\text {tot }} / l_{\text {opt }}$ are shown on Figs. 2 and 3 . The best sensitivity comes from $l_{\text {tot }} / l_{\text {opt }}$, which typically competes with the reach from $d d \mu^{+}$.

Complementarity with direct detection.-Searches for real DM scattering off nucleons (i.e., DM direct detection) can be described by the same effective operators as the ones used for the DM force; hence, we can readily compare both techniques. It is convenient to translate our DM forces bounds into equivalent exclusion regions on the spinindependent (SI) DM-nucleon scattering cross section $\sigma^{\text {SI }}$. The exclusion regions in the $\sigma^{\mathrm{SI}}-m$ plane are shown in Fig. 2. The complementarity is clear: the direct detection sensitivity always vanishes for small $m$, while our bounds from DM forces vanish at large $m$ and are valid down to zero mass. The typical exclusion regions can be conveniently classified with 


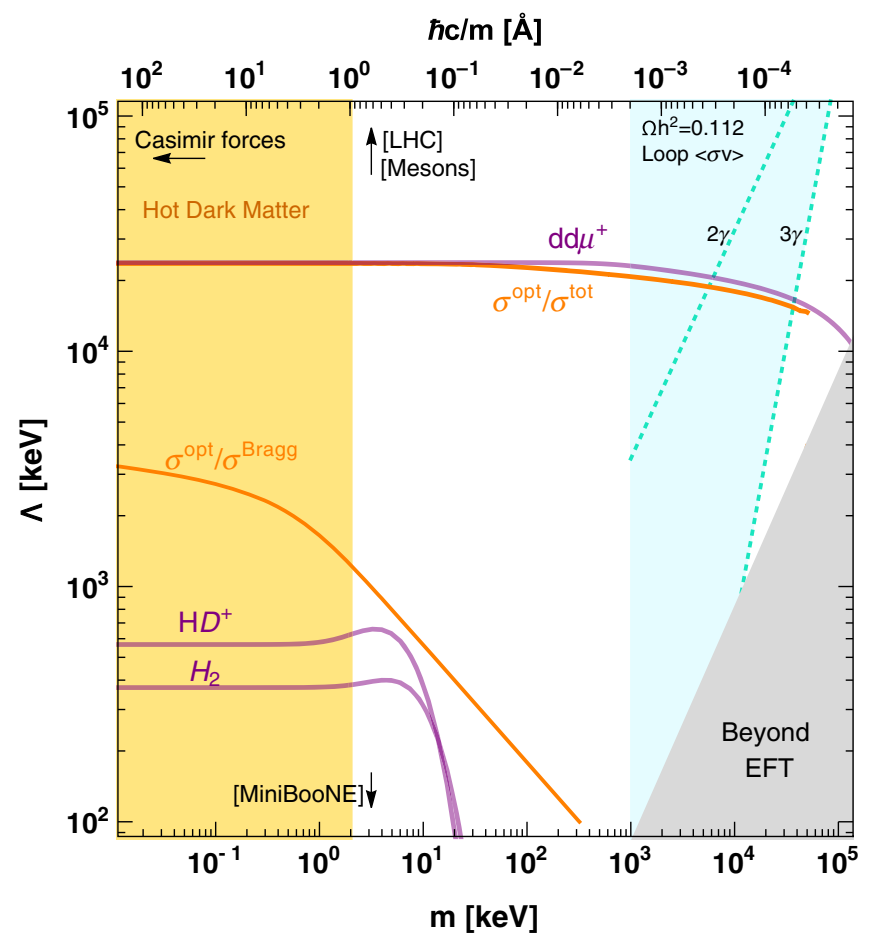

FIG. 3. Limits on the $\mathcal{O}_{b}^{1 / 2}$ interaction of Dirac DM in the $m-\Lambda$ plane. Regions excluded by precision molecular spectroscopy and neutron scattering are shown by purple and red upper boundaries, respectively. The constraint from structure formation [1-3] is shown in orange. Exclusion regions lying outside the shown parameter space (see text) are indicated with arrows. Those in brackets assume the interaction is mediated by a light leptophobic $Z^{\prime}$ with $m_{Z^{\prime}} \lesssim 10 \mathrm{MeV}, \alpha_{B} \lesssim 0.03 \alpha_{\mathrm{em}}$. We indicate the exclusion regions from Casimir force searches [68,69], from LHC missing energy searches [5], from kaon and quarkonium invisible decays [70-73] assuming flavor universal couplings, and the sensitivity region from the MiniBooNE detector $[25,26,29]$. In the gray region, the UV completion (e.g., $Z^{\prime}$ exchange) has to be specified. Dotted lines correspond to $\Omega h^{2}=$ 0.112 for thermal freeze-out with Dirac DM $\chi \bar{\chi} \rightarrow 2 \gamma, 3 \gamma$ annihilations. Blue regions correspond to phase-transition-induced freeze-out.

respect to the short-range behavior of the DM force. The $r^{-3}$, $r^{-5}$, and $r^{-7}$ regions shown in Fig. 2 are from $\mathcal{O}_{a}^{0}, \mathcal{O}_{b}^{1 / 2}$, and $\mathcal{O}_{c}^{1}$. Interestingly, the operators $\mathcal{O}_{c}^{1 / 2}$ and $\mathcal{O}_{d}^{1}$ have vanishing SI cross section, while they are probed by the DM force.

Cosmology.-There is a lot of freedom to accommodate the observed relic abundance of DM with sub-GeV masses (see, e.g., [26,28,83-85]), but in general such scenarios are independent of the DM-nucleon coupling. Here, we rather present two versions of a scenario that relies solely on the coupling to hadrons to explain the DM abundance and can be thus meaningfully confronted to our DM force boundsand to direct detection.

Loop-level freeze-out:-As DM interacts with nucleons via the operators of Eq. (1), we can assume it couples similarly to other hadrons. Whenever DM interacts with charged hadrons, DM annihilation into photons can always occur by closing the hadron loop, and thermal freeze-out is controlled by this annihilation. Annihilation is mostly into $2 \gamma$ for the scalar channel, but only into $3 \gamma$ for the vector channel. The main contribution is from charged pions. Focusing on Dirac DM annihilating as $\chi \bar{\chi} \rightarrow \gamma \gamma$ via $\mathcal{O}_{a}^{1 / 2}$ and in $\chi \bar{\chi} \rightarrow \gamma \gamma \gamma$ via $\mathcal{O}_{b}^{1 / 2}$ (possibly UV completed by a $Z^{\prime}$ ), taking the heavy pion limit and deducing the local $\gamma \gamma \gamma Z^{\prime}$ vertex from [86], we get order-of-magnitude estimates (assuming the same coupling to pion and nucleons)

$$
\langle\sigma v\rangle_{2 \gamma} \sim\left(\frac{2 \times 10^{-5}}{\mathrm{GeV}^{2}}\right) \frac{m^{4}}{\Lambda^{4}}, \quad\langle\sigma v\rangle_{3 \gamma} \sim\left(\frac{0.1}{\mathrm{GeV}^{8}}\right) \frac{m^{10}}{\Lambda^{4}} .
$$

This minimal scenario is shown in Figs. 2 and 3.

Phase-transition-induced freeze-out:- It is also possible that the DM interactions with hadrons take the form Eq. (1) only below a phase transition at $T \equiv f$. This happens, in particular, if the mediator gets a mass after phase transition, e.g., a scalar or a $Z^{\prime}$ getting mass via a dark Higgs mechanism. In such scenario, the decoupling occurs from the symmetry breaking instead of the expansion of the Universe, and the DM relic abundance depends only on $m$ and $f$. In the case of mediation by a massless species before transition, the parameter space is simply given by $\Lambda>\Lambda_{\min }$, where $\Lambda_{\min }$ is the value required for thermal freeze-out occurring in the previous scenario. We notice another intriguing realization of this mechanism: DM could actually appear at the phase transition as a result of the confinement of a strongly interacting gauge theory. It is plausible that DM Boltzmann suppression occurs during the phase transition, because chemical equilibrium should be conserved to some extent, at least for a crossover. At large $N$ and 't Hooft coupling, this scenario admits a holographic description and could be studied in this fashion.

In all the above scenarios, the annihilation into photons, if active below neutrino decoupling $(T \sim 2.3 \mathrm{MeV})$, reheats photons and tends to reduce the observed effective number of neutrinos $\left(N_{\text {eff }}\right)$ [87-89]. However, any extra relativistic species-just like the light mediators present in the second mechanism-can increase $N_{\text {eff }}$ back. For both scenarios, we require the freeze-out temperature to be above $0.1 \mathrm{MeV}$ to avoid changes in the He abundance from big bang nucleosynthesis [90], hence $m \gtrsim 1 \mathrm{MeV}$. DM annihilation into $2 \gamma$ produces $\gamma$-ray lines but is velocity suppressed for Dirac DM.

Complementarity with other experiments. - Let us finally compare the constraints from DM forces to other existing ones-apart from direct detection. From the basic assumption that DM couples to nucleons, there are constraints from Casimir force measurements and pendulum experiments, starting at $m \sim 10-100 \mathrm{eV}$. However, apart from scalar DM with the $\mathcal{O}_{a}^{0}$ operator, all the other forces are best constrained at any mass by the methods presented here [37]. 
For particle physics experiments, our low-energy EFT breaks down and comparison has to be on the basis of a specific UV completion for a given DM candidate. We focus on Dirac DM with $\mathcal{O}_{b}^{1 / 2}$ effective interaction, which is naturally UV completed by a $Z^{\prime}$ boson from a hidden $U(1)$ coupling to quark and $\chi$ currents with strength $g_{B}$. At low energy, the mapping onto $\mathcal{O}_{b}^{1 / 2}$ is given by $\Lambda^{-2}=-g_{B}^{2} m_{Z^{\prime}}^{-2}$. The $Z^{\prime}$ is leptophobic and with no kinetic mixing to the photon. We will translate constraints on the $Z^{\prime}$ model into bounds on the $\Lambda$ parameter. An important subtlety is that these translated constraints do not necessarily take the form of a lower bound on $\Lambda$, because they originate from an UV completion.

Constraint from kaon decay $K^{+} \rightarrow \pi^{+}+$invisible using the bound from [70] and the prediction from [26] [see Eq. (7)] gives $g_{B} m_{Z^{\prime}}<0.13 \mathrm{MeV}$. In the low-energy EFT, this becomes $\Lambda \lesssim 0.01 \mathrm{MeV} \alpha_{B}^{-1}$. This is an upper bound on $\Lambda$, and there is thus complementarity with the DM force, which instead sets a lower bound. A similar, subleading bound comes from $J / \Psi$ invisible decay $[28,73,91]$. The bound from kaon decay would reach down $\Lambda \sim 10 \mathrm{MeV}$ and start to compete with the DM force for $\alpha_{B} \gtrsim 0.1 \alpha_{\mathrm{em}}$. Monojet with missing energy (see, e.g., [5]) and dijet searches from the LHC are also constraining the $Z^{\prime}$ scenario. The sensitivity drops when the $Z^{\prime}$ gets light; hence, these searches put upper bounds on $\Lambda$, which, however, do not compete with the meson bounds. Finally, following Refs. [26,29], measurements at the MiniBooNE detector [25] are expected to provide an upper bound on $g_{B}$ for values of $m_{Z^{\prime}}$ down to $\sim 10-100 \mathrm{MeV}$, which implies a lower bound on $\Lambda$. For lighter $Z^{\prime}$, the sensitivity should drop, implying that the region constrained by the MiniBooNE measurements lies at smaller $\Lambda$, as indicated in Fig. 3.

In this $Z^{\prime}$ scenario, particle physics experiments do not access the region with roughly $m_{Z^{\prime}}<10 \mathrm{MeV}$ and $\alpha_{B} \lesssim$ $0.1 \alpha_{\mathrm{em}}$. The DM force measurements are probing part of this region and are thus complementary to the other experiments.

Summary.-We have calculated the quantum forces induced by sub-GeV DM coupled to nucleons, and we have shown that molecular spectroscopy and neutron scattering can be used as DM search experiments. Existing measurements put bounds on sub-GeV dark sector scenarios, with $\Lambda$ up to $O(10-100) \mathrm{MeV}$ and $m$ up to $\sim 3-50 \mathrm{MeV}$. These DM force searches are very complementary to nucleon-based direct detection. We have presented predictive cosmological scenarios that are constrained by these searches.

I thank E. Bertuzzo, G. von Gersdorff, F. Goertz, G. Grilli de Cortona, F. Iocco, E. Pontón, R. Rosenfeld, V. Sanz, and especially N. Bernal and C. S. Fong for useful discussions. I acknowledge W. G. Ubachs and G. Pignol, for providing crucial clarifications on experimental aspects, and V. Korobov for important clarifications on molecular wave functions. This work is supported by the São Paulo Research Foundation (FAPESP) under Grants No. 2011/ 11973 and No. 2014/21477-2. *sylvain@ift.unesp.br

[1] J. R. Bond and A. S. Szalay, Astrophys. J. 274, 443 (1983).

[2] M. Viel, G. D. Becker, J. S. Bolton, and M. G. Haehnelt, Phys. Rev. D 88, 043502 (2013).

[3] N. Menci, A. Grazian, M. Castellano, and N. G. Sanchez, Astrophys. J. 825, L1 (2016).

[4] T. M. Undagoitia and L. Rauch, J. Phys. G 43, 013001 (2016).

[5] V. Khachatryan et al. (CMS Collaboration), Eur. Phys. J. C 75, 235 (2015).

[6] R. Essig, J. Mardon, and T. Volansky, Phys. Rev. D 85, 076007 (2012).

[7] P. W. Graham, D. E. Kaplan, S. Rajendran, and M. T. Walters, Phys. Dark Universe 1, 32 (2012).

[8] R. Essig, M. Fernandez-Serra, J. Mardon, A. Soto, T. Volansky, and T.-T. Yu, J. High Energy Phys. 05 (2016) 046.

[9] S. K. Lee, M. Lisanti, S. Mishra-Sharma, and B. R. Safdi, Phys. Rev. D 92, 083517 (2015).

[10] Y. Hochberg, Y. Zhao, and K. M. Zurek, Phys. Rev. Lett. 116, 011301 (2016).

[11] Y. Hochberg, M. Pyle, Y. Zhao, and K. M. Zurek, J. High Energy Phys. 08 (2016) 057.

[12] W. Guo and D. N. McKinsey, Phys. Rev. D 87, 115001 (2013).

[13] K. Schutz and K. M. Zurek, Phys. Rev. Lett. 117, 121302 (2016).

[14] G. Cavoto, E. N. M. Cirillo, F. Cocina, J. Ferretti, and A. D. Polosa, Eur. Phys. J. C 76, 349 (2016).

[15] Y. Hochberg, Y. Kahn, M. Lisanti, C. G. Tully, and K. M. Zurek, Phys. Lett. B 772, 239 (2017).

[16] R. Essig, J. Mardon, O. Slone, and T. Volansky, Phys. Rev. D 95, 056011 (2017).

[17] R. Budnik, O. Chesnovsky, O. Slone, and T. Volansky, arXiv:1705.03016.

[18] S. Derenzo, R. Essig, A. Massari, A. Soto, and T.-T. Yu, Phys. Rev. D 96, 016026 (2017).

[19] R. Essig, A. Manalaysay, J. Mardon, P. Sorensen, and T. Volansky, Phys. Rev. Lett. 109, 021301 (2012).

[20] R. Essig, T. Volansky, and T.-T. Yu, Phys. Rev. D 96, 043017 (2017).

[21] C. Kouvaris and J. Pradler, Phys. Rev. Lett. 118, 031803 (2017).

[22] B. Batell, M. Pospelov, and A. Ritz, Phys. Rev. D 80, 095024 (2009).

[23] P. deNiverville, M. Pospelov, and A. Ritz, Phys. Rev. D 84, 075020 (2011).

[24] P. deNiverville, D. McKeen, and A. Ritz, Phys. Rev. D 86, 035022 (2012).

[25] R. Dharmapalan et al. (MiniBooNE Collaboration), arXiv:1211.2258.

[26] B. Batell, P. deNiverville, D. McKeen, M. Pospelov, and A. Ritz, Phys. Rev. D 90, 115014 (2014).

[27] D. E. Soper, M. Spannowsky, C. J. Wallace, and T. M. P. Tait, Phys. Rev. D 90, 115005 (2014).

[28] B. A. Dobrescu and C. Frugiuele, J. High Energy Phys. 02 (2015) 019.

[29] C. Frugiuele, Phys. Rev. D 96, 015029 (2017).

[30] S. Alekhin et al., Rept. Prog. Phys. 79, 124201 (2016).

[31] S. Fichet, J. High Energy Phys. 04 (2017) 088. 
[32] Fundamental Physics at the Intensity Frontier, https:// inspirehep.net/record/1114323.

[33] C. Frugiuele, E. Fuchs, G. Perez, and M. Schlaffer, Phys. Rev. D 96, 015011 (2017).

[34] C. Delaunay, R. Ozeri, G. Perez, and Y. Soreq, Phys. Rev. D 96, 093001 (2017).

[35] C. Delaunay and Y. Soreq, arXiv:1602.04838.

[36] J. C. Berengut et al., arXiv:1704.05068 [Phys. Rev. Lett. (to be published)].

[37] P. Brax, S. Fichet, and G. Pignol, Bounding quantum dark forces (to be published).

[38] A. V. Manohar, Lect. Notes Phys. 479, 311 (1997).

[39] M. Niu, E. Salumbides, G. Dickenson, K. Eikema, and W. Ubachs, J. Mol. Spectrosc. 300, 44 (2014).

[40] J. Biesheuvel, J. P. Karr, L. Hilico, K. S. E. Eikema, W. Ubachs, and J. C. J. Koelemeij, Nat. Commun. 7, 10385 (2016).

[41] J. Biesheuvel, J.-P. Karr, L. Hilico, K. S. E. Eikema, W. Ubachs, and J. C. J. Koelemeij, Appl. Phys. B 123, 23 (2017).

[42] D. V. Balin et al., Phys. Part. Nucl. 42, 185 (2011).

[43] M. Hori, A. Soter, D. Barna, A. Dax, R. Hayano, S. Friedreich, B. Juhasz, T. Pask, E. Widmann, D. Horvath, L. Venturelli, and N. Zurlo, Nature (London) 475, 485 (2011).

[44] J. C. J. Koelemeij, B. Roth, A. Wicht, I. Ernsting, and S. Schiller, Phys. Rev. Lett. 98, 173002 (2007).

[45] U. Bressel, A. Borodin, J. Shen, M. Hansen, I. Ernsting, and S. Schiller, Phys. Rev. Lett. 108, 183003 (2012).

[46] J. Liu, E. J. Salumbides, U. Hollenstein, J. C. J. Koelemeij, K. S. E. Eikema, W. Ubachs, and F. Merkt, J. Chem. Phys. 130, 174306 (2009).

[47] J.-P. Karr, J. Mol. Spectrosc. 300, 37 (2014).

[48] S. Schiller, D. Bakalov, and V. I. Korobov, Phys. Rev. Lett. 113, 023004 (2014).

[49] V. I. Korobov, J. C. J. Koelemeij, L. Hilico, and J.-P. Karr, Phys. Rev. Lett. 116, 053003 (2016).

[50] V. I. Korobov, L. Hilico, and J.-P. Karr, Phys. Rev. A 89, 032511 (2014).

[51] K. Pachucki, Phys. Rev. A 76, 022106 (2007).

[52] K. Pachucki, Phys. Rev. A 82, 032509 (2010).

[53] V. I. Korobov, J. Phys. B 37, 2331 (2004).

[54] V. I. Korobov, Phys. Rev. A 74, 052506 (2006).

[55] V. I. Korobov, Phys. Rev. A 77, 042506 (2008).

[56] V. I. Korobov, Phys. Rev. A 77, 022509 (2008).

[57] K. Piszczatowski, G. Łach, M. Przybytek, J. Komasa, K. Pachucki, and B. Jeziorski, J. Chem. Theory Comput. 5, 3039 (2009).

[58] J. Komasa, K. Piszczatowski, G. Łach, M. Przybytek, B. Jeziorski, and K. Pachucki, J. Chem. Theory Comput. 7, 3105 (2011).

[59] E. J. Salumbides, J. C. J. Koelemeij, J. Komasa, K. Pachucki, K. S. E. Eikema, and W. Ubachs, Phys. Rev. D 87, 112008 (2013).

[60] E. J. Salumbides, W. Ubachs, and V. I. Korobov, J. Mol. Spectrosc. 300, 65 (2014).

[61] E. J. Salumbides, A. N. Schellekens, B. Gato-Rivera, and W. Ubachs, New J. Phys. 17, 033015 (2015).

[62] W. Ubachs, J. Koelemeij, K. Eikema, and E. Salumbides, J. Mol. Spectrosc. 320, 1 (2016).
[63] V. Korobov (private communication).

[64] For $d d \mu^{+}$, the predicted energy shifts from the dark particle can also have some UV sensitivity; see [37].

[65] A. L. Erickcek, P. J. Steinhardt, D. McCammon, and P. C. McGuire, Phys. Rev. D 76, 042007 (2007).

[66] J. H. Davis, Phys. Rev. Lett. 119, 211302 (2017).

[67] G. Angloher et al. (CRESST Collaboration), Eur. Phys. J. C 76, 25 (2016).

[68] S. K. Lamoreaux, Phys. Rev. Lett. 78, 5 (1997); 81, 5475(E) (1998).

[69] J. Jaeckel and A. Ringwald, Annu. Rev. Nucl. Part. Sci. 60, 405 (2010).

[70] A. V. Artamonov et al. (BNL-E949 Collaboration), Phys. Rev. D 79, 092004 (2009).

[71] O. Tajima et al. (Belle Collaboration), Phys. Rev. Lett. 98, 132001 (2007).

[72] B. Aubert et al. (BABAR Collaboration), Phys. Rev. Lett. 103, 251801 (2009).

[73] M. Ablikim et al. (BES Collaboration), Phys. Rev. Lett. 100, 192001 (2008).

[74] V. V. Nesvizhevsky and K. V. Protasov, Classical Quantum Gravity 21, 4557 (2004).

[75] H. Leeb and J. Schmiedmayer, Phys. Rev. Lett. 68, 1472 (1992).

[76] A. Frank, P. van Isacker, and J. Gomez-Camacho, Phys. Lett. B 582, 15 (2004).

[77] P. J. S. Watson, arXiv:hep-ph/0406308.

[78] G. L. Greene and V. P. Gudkov, Phys. Rev. C 75, 015501 (2007).

[79] S. Baessler, V. V. Nesvizhevsky, K. V. Protasov, and A. Y. Voronin, Phys. Rev. D 75, 075006 (2007).

[80] V. V. Nesvizhevsky, G. Pignol, and K. V. Protasov, Phys. Rev. D 77, 034020 (2008).

[81] Y. Kamiya, K. Itagaki, M. Tani, G. N. Kim, and S. Komamiya, Phys. Rev. Lett. 114, 161101 (2015).

[82] L. Koester, H. Rauch, and E. SeymannAt. Data Nucl. Data Tables 49, 65 (1991).

[83] E. Kuflik, M. Perelstein, N. Rey-Le Lorier, and Y.-D. Tsai, Phys. Rev. Lett. 116, 221302 (2016).

[84] N. Bernal, X. Chu, C. Garcia-Cely, T. Hambye, and B. Zaldivar, J. Cosmol. Astropart. Phys. 03 (2016) 018.

[85] N. Bernal and X. Chu, J. Cosmol. Astropart. Phys. 01 (2016) 006.

[86] C. Baldenegro, S. Fichet, G. von Gersdorff, and C. Royon, J. High Energy Phys. 06 (2017) 142.

[87] C. Boehm, M. J. Dolan, and C. McCabe, J. Cosmol. Astropart. Phys. 08 (2013) 041.

[88] K. M. Nollett and G. Steigman, Phys. Rev. D 89, 083508 (2014).

[89] K. M. Nollett and G. Steigman, Phys. Rev. D 91, 083505 (2015).

[90] M. Pospelov and J. Pradler, Annu. Rev. Nucl. Part. Sci. 60, 539 (2010).

[91] N. Fernandez, J. Kumar, I. Seong, and P. Stengel, Phys. Rev. D 90, 015029 (2014).

[92] See Supplemental Material at http://link.aps.org/ supplemental/10.1103/PhysRevLett.120.131801 for more details. 